\title{
THE INITIAL-VALUE PROBLEM \\ FOR THE KELVIN-HELMHOLTZ INSTABILITIES OF HIGH-VELOCITY MAGNETIZED SHEAR LAYERS WITH GENERALIZED POLYTROPE LAWS
}

\author{
BY \\ KEVIN G. BROWN AND S. ROY CHOUDHURY \\ Department of Mathematics, University of Central Florida, Orlando, FL
}

\begin{abstract}
The general initial-value problem for the linear Kelvin-Helmholtz instability of arbitrarily compressible magnetized anisotropic velocity shear layers is considered. The time evolution of the physical quantities characterizing the layer is treated using Laplace transform techniques. Singularity analysis of the resulting equations using Fuchs-Frobenius theory yields the large-time asymptotic solutions. Since all the singular points turned out to be real, the instability is found to remain, within the linear theory, of the translationally convective shear type. No onset of rotational or vortex motion, i.e., formation of "coherent structures" occurs because there are no imaginary singularities.
\end{abstract}

1. Introduction. The Kelvin-Helmholtz instability caused by tangential velocity shear in homogeneous fluids and plasmas is of interest in investigating a variety of space, astrophysical, and geophysical situations involving sheared plasma flows. Configurations, where it is relevant, include the interface between the solar wind and the magnetosphere (Sen, 1965; Southwood, 1968; Southwood, 1974; Bridge et al., 1979; Ness et al., 1981; Pu and Kivelson, 1983), coronal streamers moving through the solar wind, the boundaries between adjacent sectors in the solar wind (Parker, 1963; Sturrock and Hartle, 1966; Jokipii and Davis, 1969), the structure of the tails of comets (Dobrowolny and D'Angelo, 1972; Ershkovich et al., 1972; Ershkovich and Chernikov, 1973; Brandt and Mendis, 1979), and the boundaries of the jets propagating from the nuclei of extragalactic double radio sources into their lobes (Turland and Scheuer, 1976; Blanford and Pringle, 1976; Begelman et al., 1984).

Early investigations of the Kelvin-Helmholtz instability were concerned with the instability caused by a tangential velocity discontinuity or jump (or vortex sheet) in incompressible and compressible fluids and plasmas (Landau, 1944; Fejer, 1964; Sen, 1964; Miles, 1957; Gerwin, 1968). Note that Fejer and Sen come to opposite conclusions on

Received June 16, 1999 and, in revised form, October 19, 1999.

2000 Mathematics Subject Classification. Primary 76E25, 76E20.

E-mail address: choudhur@longwood.cs.ucf.edu 
the stabilizing effect of the compressibility; according to Gerwin, "this discrepancy is apparently not resolved".

The unmagnetized vortex sheet is found to be unstable at all wavenumbers for modes sufficiently transverse to the zero-order flow, or for modes along the flow with Mach number less than $2 \sqrt{2}$. However, as shown by Syrovatskii (1957) (also see Landau and Lifshitz (1984)), there always exist propagation directions $\bar{k}$ along which the perturbations are unstable.

In the presence of a magnetic field parallel to the flow the instability of the incompressible vortex sheet is completely stabilized unless the velocity discontinuity exceeds twice the Alfvén speed. Here, the pioneering contributions were by Syrovatskii (1957).

Lerche (1966) emphasized the importance of considering the finite thickness of the shear layer. The linear Kelvin-Helmholtz instability of shear layers (a region of finite width over which the velocity change occurs) for flows with a subsonic velocity change was considered by Chandrasekhar (1981). An incompressible shear layer having a "hyperbolic tangent" profile was considered by Michalke (1964). For a wave number $k$ and a shear layer width $L$, he found a criterion $k L<2$ for instability, so that short wavelength modes were stabilized for the finite width velocity shear. The stability characteristics of finite width unmagnetized shear layers have been considered by several authors (Blumen, 1970; Blumen et al., 1975; Ray, 1982; Miura and Pritchett, 1982; Roy Choudhury and Lovelace, 1986). The finite width shear layers exhibit unstable traveling wave modes satisfying radiation boundary conditions. These modes are absent for the unmagnetized vortex sheet, and present for the magnetized vortex sheet in a very small range of Mach numbers. The presence of the traveling wave modes means that the finite width layer is unstable at all Mach numbers. In addition, standing wave solutions analogous to the "warping" modes which occur for the vortex sheet are also present at long wavelengths and small values of the Mach number. Magnetized shear layers described by the MHD formalism have been considered for a linear velocity profile layer (Roy Choudhury, 1986; Ray and Ershkovich, 1983; Roy Choudhury and Lovelace, 1986) with both standing and traveling wave solutions, and for a hyperbolic tangent velocity profile (Miura and Pritchett, 1982) for only standing wave modes. A magnetic field parallel to the flow is found to stabilize both classes of modes. Computer simulation studies of the KelvinHelmholtz instabilities of planar, magnetized shear layers (Nepveu, 1980; Tajima and Leboeuf, 1980; Miura and Pritchett, 1982; Pritchett and Coroniti, 1984; Miura, 1984) and of cylindrical axisymmetric jets (Norman et al., 1982) have also been carried out. Other recent work has been reviewed by Larosa and Moore (1993).

In the above studies, most of the treatments used the collision-dominated hydromagnetic equations with scalar gas pressure approximation. The scalar gas pressure approximation is not appropriate in dilute plasmas such as the coronal streamers and the solar wind. The plasma in the interplanetary medium, earth's magnetosphere and the polar exosphere are collisionless. There is a transition zone in solar wind where plasma is neither fully collisional nor collisionless. The Kelvin-Helmholtz instability has been discussed in anisotropic plasma using Chew, Goldberger, and Low (CGL) equations for the situations where collisions are not sufficiently strong to keep the pressure a scalar but sufficiently strong to prevent the heat flow and other transport processes. Roy Choudhury and Patel 
(1985) have considered the Kelvin-Helmholtz instability of an anisotropic, finite width, supersonic shear layer and investigated the nonlocal coupling of the firehose and mirror instabilities via a spatially varying velocity. Duhau et al. $(1970,1971)$ have discussed the problem of a tangential velocity discontinuity in a collisionless hydromagnetic region using CGL approximation. Duhau and Gratton (1972) have investigated the effect of compressibility on the stability of a vortex sheet in an ideal magnetofluid. Rajaram et al. $(1978,1980)$ have examined the contact discontinuities on two collisionless fluids in the magnetosphere across the cusp region of the solar wind magnetosphere boundary. Talwar $(1964,1965)$ has presented a study of Kelvin-Helmholtz instability of two streams of homogeneous anisotropic plasma. $\mathrm{Pu}$ (1989) has developed a new approach called the drift kinetic approximation (DKA) for collisionless space plasmas.

However, more general anisotropic models than the CGL model are necessary in treating low collision regimes, such as in the transitional region of the solar wind. In this connection, we employ the plasma model with generalized polytrope laws for the parallel and perpendicular pressures. This model, proposed by Abraham-Shrauner (1973), uses double adiabatic pressure laws with generalized polytrope indices together with the other magnetohydrodynamic (MHD) equations. For specific choices of polytrope indices, it contains both the MHD and CGL models as special cases. The Kelvin-Helmholtz instability of anisotropic high-velocity shear layers has been considered recently by us using this model (Brown and Roy Choudhury, 1998).

In this paper we study the general initial-value problem for the linear Kelvin-Helmholtz instability of arbitrarily compressible magnetized velocity shear layers with general anisotropy via the use of Laplace transforms. To our knowledge, this has not been studied previously. The simplest example of the solution of the general initial-value problem for linear stability analysis of a fluid flow has been given by Case (1960a, b). Our treatment will follow that analysis to obtain the time-asymptotic solutions for this instability. The qualitative long-time features of the instability are deduced from these solutions. In particular, we find that there is no occurrence of rotational motion, i.e., the instability remains of the shear or translationally convective type.

The remainder of this paper is organized as follows. Section 2 describes the initialvalue problem, with some of the mathematical details being contained in the Appendix. In Section 3 we obtain the long-time limit of the solutions, and discuss its implications for the behavior of the unstable modes.

2. The initial-value problem. The equations for a compressible, inviscid, infinitely conducting plasma are:

$$
\begin{gathered}
\rho \frac{d \vec{v}}{d t}=-\vec{\nabla} \cdot \overrightarrow{\mathbf{p}}+\frac{1}{4 \pi}(\vec{\nabla} \times \overrightarrow{\mathbf{B}}) \times \overrightarrow{\mathbf{B}} \\
\frac{\partial \rho}{\partial t}=-\vec{\nabla} \cdot(\rho \vec{v}) \\
\frac{\partial \vec{B}}{\partial t}=\vec{\nabla} \times(\vec{v} \times \overrightarrow{\mathbf{B}})
\end{gathered}
$$




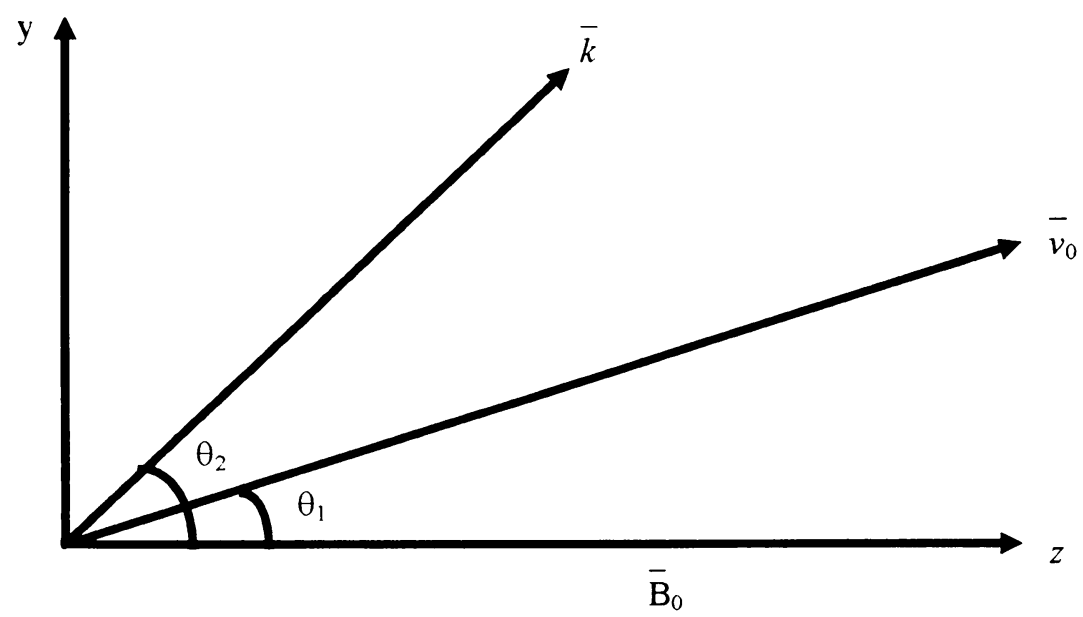

FIG. 1. Geometry of the equilibrium configuration and flow for the compressible tangential velocity discontinuity. The wave vector $\bar{k}$ for the perturbation quantities is shown. Perturbations $\pm \theta_{2}$ are degenerate.

$$
\begin{gathered}
\vec{\nabla} \cdot \vec{B}=0 \\
\overrightarrow{\mathbf{p}}=\mathbf{p}_{\perp} \overrightarrow{\mathbf{I}}+\left(\mathbf{p}_{\|}-\mathbf{p}_{\perp}\right) \hat{n} \hat{n} \\
\overrightarrow{\mathbf{I}}=\text { unit dyadic }
\end{gathered}
$$

and the adiabatic equations of state with generalized polytrope exponents $\alpha, \beta, \epsilon$, and $\gamma$ are (Abraham-Shrauner, 1973):

$$
\frac{d}{d t}\left(\frac{\mathbf{p}_{\|} B^{\alpha}}{\rho^{\gamma}}\right)=0
$$

and

$$
\frac{d}{d t}\left(\frac{\mathbf{p}_{\perp}}{\rho^{\epsilon} B^{\beta}}\right)=0 .
$$

Here, $\|$ and $\perp$ denote components parallel and perpendicular to the magnetic field respectively and $\frac{d}{d t}=\left(\frac{\partial}{\partial t}+\vec{v} \cdot \vec{\nabla}\right)$. For the special choices (a) $\alpha=0, \beta=0, \epsilon=\frac{5}{3}$, $\gamma=\frac{5}{3}$, and $\frac{p_{\|}}{p_{\perp}}=1$, and (b) $\alpha=2, \beta=1, \epsilon=1, \gamma=3$, this model reduces to the MHD and CGL models respectively. The equilibrium we consider (Fig. 1) has a flow velocity $v_{0}(x)=v_{0 y}(x) \hat{y}+v_{0 z}(x) \hat{z}$, a uniform magnetic field $\mathbf{B}=B_{0} \hat{z}$, constant density $\rho$, and pressures $p_{\|}$and $p_{\perp}$.

The first order perturbation quantities are of the form $f(x) \exp \left[i\left(k_{y} y+k_{z} z-\omega t\right)\right]$. The frequency $\omega$ is assumed to have at least a small positive imaginary part, so that the solutions correspond to those of an initial-value problem. Linearizing equations (1), we obtain

$$
i \Omega \delta \rho=-\rho\left(\delta v_{x}^{\prime}+i k_{y} \delta v_{y}+i k_{z} \delta v_{z}\right)
$$




$$
\begin{gathered}
i \Omega \rho \delta v_{z}+i k_{z} \delta p_{\|}+\left(p_{\perp}-p_{\|}\right) i k_{z} \frac{\delta B_{z}}{B_{0}}=0 \\
i \Omega \rho \delta v_{x}+\delta p_{\perp}^{\prime}+\frac{B_{0}}{4 \pi} \delta B_{z}^{\prime}-\frac{i k_{z} B_{0}}{4 \pi} \delta B_{x}\left(\frac{p_{\perp}-p_{\|}}{B_{0}^{2} / 4 \pi}+1\right)=0 \\
i \Omega \rho \delta v_{y}+i k_{y} \delta p_{\perp}+\frac{i k_{y} B_{0}}{4 \pi} \delta B_{z}-\frac{i k_{z} B_{0}}{4 \pi} \delta B_{y}\left(\frac{p_{\perp}-p_{\|}}{B_{0}^{2} / 4 \pi}+1\right)=0 \\
\frac{\delta p_{\perp}}{p_{\perp}}=\epsilon \frac{\delta \rho}{\rho}+\beta \frac{\delta B_{z}}{B_{0}} \\
\frac{\delta p_{\|}}{p_{\|}}=\gamma \frac{\delta \rho}{\rho}-\alpha \frac{\delta B_{z}}{B_{0}} \\
i \Omega \delta B_{x}=i k_{z} B_{0} \delta v_{x} \\
i \Omega \delta B_{y}=i k_{z} B_{0} \delta v_{y}+S v_{0}^{\prime} \delta B_{x} \\
i \Omega \delta B_{z}=i k_{z} B_{0} \delta v_{z}+C v_{0}^{\prime} \delta B_{x}-B_{0}\left[\delta v_{x}^{\prime}+i k_{y} \delta v_{y}+i k_{z} \delta v_{z}\right] \\
\delta B_{x}^{\prime}+i k_{y} \delta B_{y}+i k_{z} \delta B_{z}=0
\end{gathered}
$$

Here the prime denotes a derivative with respect to $x, C \equiv \cos \left(\theta_{1}\right), S=\sin \left(\theta_{1}\right)$, and $\Omega \equiv\left(k_{y} v_{0} S+k_{z} v_{0} C-\omega\right)$. The equilibrium quantities are $p_{\|}, p_{\perp}, \rho$, and $v_{0}(x)$.

Given the value of $\delta v_{x}(x, t=0), \delta v_{y}(x, t=0), \delta v_{z}(x, t=0), \delta p_{\|}(x, t=0), \delta p_{\perp}(x, t=$ $0), \delta \rho(x, t=0), \delta B_{x}(x, t=0), \delta B_{y}(x, t=0)$, and $\delta B_{z}(x, t=0)$, we require the solution for all $t>0$. This is the initial-value problem we now solve.

Defining the Laplace transform of a variable $\delta \gamma(x, t)$ as

$$
\Gamma(x, s) \equiv \int_{0}^{\infty} e^{-s t} \delta \gamma(x, t) \dot{d} t
$$

by the capital symbol, some of equations (2) become

$$
\begin{aligned}
& i \rho\left(k_{y} v_{0 y}+k_{z} v_{0 z}\right) V_{z}+\rho\left[s V_{z}-\delta v_{z}(x, t=0)\right]+i k_{z} P_{\|}+\left(\frac{p_{\perp}-p_{\|}}{B_{0}}\right) i k_{z} B_{z}=0 \\
& i \rho\left(k_{y} v_{0 y}+k_{z} v_{0 z}\right) V_{x}+\rho\left[s V_{x}-\delta v_{x}(x, t=0)\right]+P_{\perp}^{\prime}+\frac{B_{0}}{4 \pi} B_{z}^{\prime} \\
& -\frac{i k_{z} B_{0}}{4 \pi}\left(\frac{p_{\perp}-p_{\|}}{B_{0}^{2} / 4 \pi}+1\right) B_{x}=0 \\
& i \rho\left(k_{y} v_{0 y}+k_{z} v_{0 z}\right) V_{y}+\rho\left[s V_{y}-\delta v_{y}(x, t=0)\right]+i k_{y} P_{\perp}+\frac{i k_{y} B_{0}}{4 \pi} B_{z} \\
& -\frac{i k_{z} B_{0}}{4 \pi}\left(\frac{p_{\perp}-p_{\|}}{B_{0}^{2} / 4 \pi}+1\right) B_{y}=0 \\
& i\left(k_{y} v_{0 y}+k_{z} v_{0 z}\right) B_{x}+\left[s B_{x}-\delta B_{x}(x, t=0)\right]=i k_{z} B_{0} V_{x} \\
& i\left(k_{y} v_{0 y}+k_{z} v_{0 z}\right) B_{y}+\left[s B_{y}-\delta B_{y}(x, t=0)\right]=i k_{z} B_{0} V_{y}+v_{0 y}^{\prime} B_{x}
\end{aligned}
$$




$$
\begin{aligned}
i\left(k_{y} v_{0 y}+k_{z} v_{0 z}\right) B_{z}+\left[s B_{z}-\delta B_{z}(x, t=0)\right] & =i k_{z} B_{0} V_{z}+v_{0 z}^{\prime} B_{x} \\
& -B_{0}\left[V_{x}^{\prime}+i k_{y} V_{y}+i k_{z} V_{z}\right] .
\end{aligned}
$$

These may be combined into a composite equation for $B_{x}$ with the dimensionless flow velocity given by

$$
u_{D}=\frac{k_{y} v_{0 y}+k_{z} v_{0 z}-i s}{k_{z} S_{\perp}} .
$$

Given the initial data, the solutions of this composite equation will be unique except at discrete values of $s$, which are the eigenvalues and for which $B_{x}$ satisfies the homogeneous part of the composite equation. The solution of the composite equation as $s$ approaches any eigenvalue will have the solution for $B_{x}$ containing a simple pole in $x$ (for all $x$ ) (Case, 1960; Kaup, 1990). The solutions of the homogeneous problem with radiative boundary conditions have been considered earlier by Roy Choudhury and Lovelace (1984).

The solution of the composite equation also has other singularities in $s$. These occur at the singular points of the equation and depend on $x$. These will be found to also contribute to the time-asymptotic solution. In order to consider these, it will be convenient to return to (2). Notice that the assumed time dependence $e^{-i \omega t}$ in these equations corresponds to Fourier transforming in time (Krall and Trivelpiece, 1973). We will consider (2) and later obtain the correspondence with the more standard Laplace transform in time above by setting

$$
\omega \equiv i s
$$

so that (5) yields

$$
u_{D}=\frac{k_{y} v_{0 y}+k_{z} v_{0 z}-i s}{k_{z} S_{\perp}}=\frac{k_{y} v_{0 y}+k_{z} v_{0 z}-\omega}{k_{z} S_{\perp}} .
$$

Using equations (2) (with $e^{-i \omega t}$ time dependence), we obtain the composite equation (Brown and Roy Choudhury, 1997):

$$
\begin{aligned}
\frac{1}{K_{z}^{2}}[ & \frac{i \epsilon C M u_{D} \delta b_{x}}{\left(u_{D}^{2}-\gamma r^{2}\right)}-\left\{\frac{\epsilon u_{D}^{2}-(1+\alpha) r^{2} \epsilon+\epsilon}{\left(u_{D}^{2}-\gamma r^{2}\right)}+\beta\right\} \\
& \left.\cdot\left\{\frac{\left(1-r^{2}+q_{D}^{2}-u_{D}^{2}\right)}{\left[T^{2}\left(-\epsilon \Gamma_{1}+i \beta+i q_{D}^{2}\right)+i\left(1-r^{2}+q_{D}^{2}-u_{D}^{2}\right)\right]}\right\}\left(\delta b_{x}^{\prime}+i K_{y} \chi_{4} \delta b_{x}\right)\right]^{\prime} \\
& -\frac{q_{D}^{2}}{K_{z}^{2}}\left[\left\{\frac{\left(1-r^{2}+q_{D}^{2}-u_{D}^{2}\right)}{\left[T^{2}\left(-\epsilon \Gamma_{1}+i \beta+i q_{D}^{2}\right)+i\left(1-r^{2}+q_{D}^{2}-u_{D}^{2}\right)\right]}\right\}\left(\delta b_{x}^{\prime}+i K_{y} \chi_{4} \delta b_{x}\right)\right]^{\prime} \\
& -i\left(1-r^{2}+q_{D}^{2}-u_{D}^{2}\right) \delta b_{x}=0
\end{aligned}
$$

for the $x$-component of the magnetic field, where

$$
\left(\delta b_{x}^{\prime}+i K_{y} \chi_{4} \delta b_{x}\right)=\left(\delta b_{x}^{\prime}-T\left[\frac{\epsilon T\left(\frac{C M u_{D}}{u_{D}^{2}-\gamma r^{2}}\right)+S M u_{D}}{1-r^{2}+q_{D}^{2}-u_{D}^{2}}\right] \delta b_{x}\right)
$$

and

$$
\Gamma_{1}=\frac{i\left\{(1+\alpha) r^{2}-1\right\}-i u_{D}^{2}}{u_{D}^{2}-\gamma r^{2}}
$$


We also define the dimensionless anisotropy parameter

$$
r=\frac{S_{\|}}{S_{\perp}}
$$

and the ratio of the magnetic field-energy density to the perpendicular thermal energy density (the inverse plasma beta)

$$
q_{D}^{2} \equiv\left(\frac{v_{A}}{S_{\perp}}\right)^{2}
$$

where the Alfvén speed is

$$
v_{A}^{2} \equiv \frac{B_{0}^{2}}{4 \pi \rho} .
$$

We make use of the dimensionless wave numbers in the $y$ and $z$ directions, $K_{y}$ and $K_{z}$ respectively, and $T=\tan \theta_{2}$. The Mach number of the anisotropic vortex sheet is

$$
\left.M \equiv \text { (velocity discontinuity } / S_{\perp}\right)=\left(\frac{2 v_{0}}{S_{\perp}}\right)
$$

and

$$
\frac{\delta B_{x}}{B_{0}} \equiv \delta b_{x}
$$

We have also made use of

$$
\chi_{4}=\frac{\left[-\frac{\epsilon k_{y} k_{z} B_{0} p_{\perp} \chi_{2}}{\Omega}-\rho \Omega S v_{0}^{\prime}\right]}{\chi_{6}},
$$

where

$$
\begin{aligned}
& \chi_{6}=i k_{z}^{2}\left(p_{\perp}-p_{\|}+\frac{B_{0}^{2}}{4 \pi}\right)-i \rho \Omega^{2} \\
& \chi_{2}=\rho \Omega C v_{0}^{\prime}\left[\rho \Omega B_{0}-\frac{\gamma k_{z}^{2} B_{0} p_{\|}}{\Omega}\right]^{-1} .
\end{aligned}
$$

Equation (7) has three singular points,

$$
u_{D}=r \sqrt{\gamma}
$$

and

$$
u_{D}=\frac{\sqrt{C_{1} \pm \sqrt{C_{2}}}}{\sqrt{2}}
$$

where

$$
\begin{gathered}
C_{1}=1+q_{D}^{2}-r^{2}+\gamma r^{2}+\left(\beta+\epsilon+q_{D}^{2}\right) T^{2}, \\
C_{2}=\left[-1-q_{D}^{2}+r^{2}-\gamma r^{2}-\left(\beta+\epsilon+q_{D}^{2}\right) T^{2}\right]^{2} \\
-4\left[\gamma r^{2}+\gamma q_{D}^{2} r^{2}-\gamma r^{4}-\epsilon T^{2}+\epsilon r^{2} T^{2}+\alpha \epsilon r^{2} T^{2}+\beta \gamma r^{2} T^{2}+\gamma q_{D}^{2} r^{2} T^{2}\right] .
\end{gathered}
$$

The solutions near these singular points will be necessary later for the derivation of the time-asymptotic solution. This solution, and its somewhat lengthy derivation, is contained in Appendix A. 
Having described the Laplace transform of the general solution, we next proceed to the long-time solutions.

3. Time-asymptotic solutions and discussion. In this section, we look at the long-time behavior of the solutions of equation (7). Note that we used a Fourier transform in Section 2 (all perturbation quantities were of the form $e^{-i \omega t}$ ). Hence, to consider the correspondence with the more standard Laplace transform in time, we make the replacement $(6 \mathrm{a})$ or, in the moving frame,

$$
u_{D}=\frac{k_{y} v_{0 y}+k_{z} v_{0 z}-i s}{k_{z} S_{\perp}}=-\frac{i}{k_{z} S_{\perp}}\left(s+i k_{y} v_{0 y}+i k_{z} v_{0 z}\right) \text {. }
$$

Thus, small values of $u_{D}$ correspond to small $s$ and, hence, to long-time asymptotic solutions. Similarly, around a singularity at $u_{D}=\eta$ the dominant terms in powers of $\left(u_{D}-\eta\right)$ around the singularity govern the long-time asymptotic behavior from that solution.

From equations (A13) and (A14), the general solution of the fluid-dynamical magnetic field perturbation (in the $x$-direction) equation (7) around the singularity $u_{D}=r \sqrt{\gamma}$ is

$$
\begin{aligned}
\delta b_{x}=\kappa_{0} & {\left[1-\frac{K_{z}^{2} \lambda_{1} T^{2}}{\lambda_{2}\left(2 \lambda_{1} \lambda_{2}+\epsilon C M T^{2}\right)} x^{2}+O\left(x^{4}\right)\right] } \\
& +\kappa_{2}\left[x^{1-\frac{\epsilon C M T^{2}}{2 \lambda_{1} \lambda_{2}}}+\frac{\epsilon C M r \sqrt{\gamma}\left(\lambda_{1}+\beta T^{2}\right)\left(\epsilon C M T^{2}-2 \lambda_{1} \lambda_{2}\right)}{\lambda_{1} \lambda_{2} \lambda_{3}\left(4 \lambda_{1} \lambda_{2}-\epsilon C M T^{2}\right)} x^{2-\frac{\epsilon C M T^{2}}{2 \lambda_{1} \lambda_{2}}}\right],
\end{aligned}
$$

where

$$
\begin{aligned}
& \lambda_{1}=1-r^{2}+q_{D}^{2}-\gamma r^{2}, \quad \lambda_{2}=T M S+M C, \\
& \lambda_{3}=\epsilon\left((\gamma-1-\alpha) r^{2}+1\right), \quad x=\left(u_{D}-r \sqrt{\gamma}\right) .
\end{aligned}
$$

Using equations (2) and (11a) yields

$$
\begin{aligned}
\delta b_{y}(s) & =\text { constant } \\
\delta b_{z}(s) & =\text { constant } \\
\delta v_{x}(s) & =\text { constant } \cdot \phi \\
\delta v_{y}(s) & =\text { constant } \\
\delta v_{z}(s) & =\text { constant } / \phi
\end{aligned}
$$

where

$$
\phi \equiv s+i k_{y} v_{0 y}(x)+i k_{z} v_{0 z}(x) .
$$

There are two basic contributions to the solution of the initial-value problem: (i) contours around the eigenvalues and (ii) for any fixed value of $x$, there will be a regular singular point of the differential system (7).

The contribution from the eigenvalues will be a growing (global) eigenmode with a frequency of $\operatorname{Im}\left(s_{j}\right)$ and a growth rate of $\operatorname{Re}\left(s_{j}\right)$, where $s_{j}$ is the eigenvalue. This contribution has been very well described in the literature (Roy Choudhury and Lovelace, 1984, 1986; Miura and Pritchett, 1982).

On the other hand, the pole singularities can also give a contribution. For example, (11f) has a pole of order 1 about $\phi=0$. Such a pole would give a nonzero contribution. 
Using the identity (10), and noting that the dominant long-time asymptotic behavior is obtained from the dominant small- $u_{D}$ behavior in the Laplace transformed domain, we may obtain the time-asymptotic behavior of the fluid-dynamical velocity shear layer by inverse-Laplace transforming (11). Letting $\alpha(s, x)$ be analytic in $s$ near $r=0$, one may straightforwardly show that for large times (Kaup, 1990),

$$
\begin{gathered}
\frac{1}{2 \pi i} \int_{R-i \infty}^{R+i \infty} \frac{\alpha(s, x)}{\phi^{2}(s, x)} e^{s t} d s=t e^{-i\left(k_{y} v_{0 y}(x)+k_{z} v_{0 z}(x)\right) t} \alpha\left(-i\left(k_{y} v_{0 y}(x)+i k_{z} v_{0 z}(x)\right), x\right), \\
\frac{1}{2 \pi i} \int_{R-i \infty}^{R+i \infty} \frac{\alpha(s, x)}{\phi(s, x)} e^{s t} d s=e^{-i\left(k_{y} v_{0 y}(x)+k_{z} v_{0 z}(x)\right) t} \alpha\left(-i\left(k_{y} v_{0 y}(x)+i k_{z} v_{0 z}(x)\right), x\right), \\
\frac{1}{2 \pi i} \int_{R-i \infty}^{R+i \infty} \alpha(\beta, x) e^{s t}(\ln \phi) d s \rightarrow 0,
\end{gathered}
$$

where $R$ is chosen so that the contour is to the right of all singularities in the complex $s$-plane.

Using standard Laplace inversion formulae, (11a) yields

$$
\delta b_{x}(t) \sim \delta(t) .
$$

Next, using (11b)-(11f) and (13) the long-time asymptotic behavior of the magnetic field and velocity perturbations is

$$
\begin{gathered}
\delta b_{y}(t) \sim \delta(t), \\
\delta b_{z}(t) \sim \delta(t), \\
\delta v_{x}(t) \sim \delta^{\prime}(t)+i\left(k_{y} v_{0 y}(x)+k_{z} v_{0 z}(x)\right) \delta(t), \\
\delta v_{y}(t) \sim \delta(t), \\
\delta v_{z}(t) \sim e^{i\left(k_{y} v_{0 y}(x)+k_{z} v_{0 z}(x)\right) t}
\end{gathered}
$$

where the $y$ and $z$ dependencies have been included. From these equations we may conclude that the linear fields die away to zero, except the $x$ and $z$ velocities approach constant amplitudes and propagate in the $y$ and $z$ directions with phase-speeds $v_{0 y}(x)$ and $v_{0 z}(x)$ respectively. Thus, considering the pole contribution from the singularity $u_{D}=r \sqrt{\gamma}$ (Kaup, 1990) to the linear instability, the flow remains essentially a shear-flow, with no rotational motion, or vortex or "coherent-structure" formation in the regions of layers of fluid near the singularity. This is in contrast, for example, to the rotational vortex motion generated near the so-called "magnetron" singularity for shear-flows in non-neutral pure-electron plasmas (Kaup, 1990).

In a similar manner, we shall now investigate the behavior near the other two singularities given by $(9 \mathrm{~b})$. The behavior near these two singularities turns out to be similar and as a result we shall consider them together. For reference purposes, we shall denote these singularities in the following manner:

$$
u_{D}=\frac{\sqrt{C_{1}+\sqrt{C_{2}}}}{\sqrt{2}}=\eta_{1}
$$


and

$$
u_{D}=\frac{\sqrt{C_{1}-\sqrt{C_{2}}}}{\sqrt{2}}=\eta_{2} .
$$

Using (A9) and (A10), it is straightforward to show, following the above procedure, that the fields near these singularities have the same form as those in equation (14) (near $u_{D}=r \sqrt{\gamma}$ ). Thus, the conclusions following equation (14) hold near $u_{D}=\eta_{1,2}$ as well.

In particular, the results obtained above from the Laplace transform solution of the initial-value problem for the linear Kelvin-Helmholtz instability establish that the linear instability remains essentially a shear-instability, with translations occurring in $y$ and $z$. No rotational motion, or vortex or "coherent-structure" formation, in the regions of layers of fluid near the singularities, occurs. This is in contrast, for example, to the rotational vortex motion generated near the so-called "magnetron" singularity for shear-flows in non-neutral pure-electron plasmas (Kaup, 1990). The reason for the absence of such coherent-structure formulation in our problem is not difficult to establish mathematically. The singularities in our case occurred at $u_{D}=r \sqrt{\gamma}$ and $u_{D}=\frac{\sqrt{C_{1} \pm \sqrt{C_{2}}}}{\sqrt{2}}$ and turned out to be real. Imaginary singularities $u_{D}= \pm i \Omega$ are necessary for the establishment of vortex motion with angular frequency $\pm \Omega$ or any physical variable at large-time. Physically, too, the complex interaction of the electromagnetic fields with the non-neutral fluid (electron plasma), leading to the formation of rotating vortices, is absent for our neutral fluids. The analysis above demonstrates that this clearly precludes the onset of vortex motion in the long-time behavior of the supersonic Kelvin-Helmholtz instability, at least while the perturbation amplitudes are small enough for the linear theory to remain valid. In the nonlinear regime, it is well known that this instability leads to coherent vortex structures (Brown and Roshko, 1974; Winant and Browand, 1974; Aref and Siggia, 1981; Norman et al., 1982; Aref and Tryggvason, 1984). Note that the contribution from the eigenvalues, which have been considered earlier by normal mode analysis (Roy Choudhury and Lovelace, 1984; Miura and Pritchett, 1982), gives growing or unstable solutions which eventually become too large to be described by the linear theory.

Appendix A. Singularity analysis of magnetic field perturbation (x-direction) equation for supersonic anisotropic magnetized shear layers.

The equation for the magnetic field perturbation ( $x$-direction) was derived in Section 2 and has the form

$$
\begin{aligned}
\frac{1}{K_{z}^{2}} & {\left[\frac{i \epsilon C M u_{D} \delta b_{x}}{\left(u_{D}^{2}-\gamma r^{2}\right)}-\left\{\frac{\epsilon u_{D}^{2}-(1+\alpha) r^{2} \epsilon+\epsilon}{\left(u_{D}^{2}-\gamma r^{2}\right)}+\beta\right\}\right.} \\
& \left.\cdot\left\{\frac{\left(1-r^{2}+q_{D}^{2}-u_{D}^{2}\right)}{\left[T^{2}\left(-\epsilon \Gamma_{1}+i \beta+i q_{D}^{2}\right)+i\left(1-r^{2}+q_{D}^{2}-u_{D}^{2}\right)\right]}\right\}\left(\delta b_{x}^{\prime}+i K_{y} \chi_{4} \delta b_{x}\right)\right]^{\prime} \\
& -\frac{q_{D}^{2}}{K_{z}^{2}}\left[\left\{\frac{\left(1-r^{2}+q_{D}^{2}-u_{D}^{2}\right)}{\left[T^{2}\left(-\epsilon \Gamma_{1}+i \beta+i q_{D}^{2}\right)+i\left(1-r^{2}+q_{D}^{2}-u_{D}^{2}\right)\right]}\right\}\left(\delta b_{x}^{\prime}+i K_{y} \chi_{4} \delta b_{x}\right)\right]^{\prime} \\
& -i\left(1-r^{2}+q_{D}^{2}-u_{D}^{2}\right) \delta b_{x}=0
\end{aligned}
$$


where

$$
\begin{aligned}
\left(\delta b_{x}^{\prime}+i K_{y} \chi_{4} \delta b_{x}\right) & =\left(\delta b_{x}^{\prime}-T\left[\frac{\epsilon T\left(\frac{C M u_{D}}{u_{D}^{2}-\gamma r^{2}}\right)+S M u_{D}}{1-r^{2}+q_{D}^{2}-u_{D}^{2}}\right] \delta b_{x}\right), \\
\Gamma_{1} & =\frac{i\left\{(1+\alpha) r^{2}-1\right\}-i u_{D}^{2}}{u_{D}^{2}-\gamma r^{2}}
\end{aligned}
$$

and (Brown and Roy Choudhury, 1997)

$$
{ }^{\prime} \equiv \frac{d}{d \bar{x}}, \quad u_{D}=(T M S+M C) \bar{x}-W_{D}
$$

Here, we assume a linear velocity profile (Roy Choudhury and Lovelace, 1984; Ray, 1982). By taking the derivative of $u_{D}$ with respect to $\bar{x}$, we get $\frac{d u_{D}}{d \bar{x}}=(T M S+M C) \frac{d}{d u_{D}}=$ $\lambda_{2} \frac{d}{d u_{D}}$. Replacing $d \bar{x}$ by $\frac{d u_{D}}{\lambda_{2}}$ in (A1), equation (A1) takes the following form:

$$
\begin{gathered}
\frac{\lambda_{2}}{K_{z}^{2}}\left[\frac{\left(\frac{\chi_{2}}{\chi_{3}}+\beta+q_{D}^{2}\right) i \chi_{1} \lambda_{2}}{T^{2}\left(\frac{\chi_{2}}{\chi_{3}}+\beta+q_{D}^{2}\right)+\chi_{1}}\right] \frac{d^{2}}{d u_{D}^{2}}\left(\delta b_{x}\right) \\
+\frac{\lambda_{2}}{K_{z}^{2}}\left[\frac{-\left(\frac{\chi_{2}}{\chi_{3}}+\beta-q_{D}^{2}\right) T u_{D}\left(\epsilon C M T+S M \chi_{3}\right)}{T^{2}\left(\chi_{2}+\chi_{3}\left(\beta+q_{D}^{2}\right)\right)+\chi_{1} \chi_{3}}+\frac{i \epsilon C M u_{D}}{\chi_{3}}\right] \frac{d}{d u_{D}}\left(\delta b_{x}\right) \\
-i \chi_{1} \delta b_{x}=0
\end{gathered}
$$

where

$$
\begin{gathered}
\chi_{1}=1-r^{2}+q_{D}^{2}-u_{D}^{2}, \quad \chi_{2}=-\epsilon\left((1+\alpha) r^{2}-1-u_{D}^{2}\right), \\
\chi_{3}=u_{D}^{2}-\gamma r^{2} .
\end{gathered}
$$

This equation has singular points at $\chi_{3}=0$ or $u_{D}=r \sqrt{\gamma}$ and $T^{2}\left(\chi_{2}+\chi_{3}\left(\beta+q_{D}^{2}\right)\right)+$ $\chi_{1} \chi_{3}=0$ which give the two lengthy singularities.

We use the Fuchs-Frobenius method (Ince, 1956; Bender and Orzag, 1978) to find the solutions of (A2) about the singular points. Let $\delta b_{x}(x, \alpha)=\sum_{n=0}^{\infty} a_{n} x^{n+\alpha}$, where $\alpha$ is the indicial exponent to be determined and $x=\left(u_{D}-\eta\right)$ for a singularity $\eta$. When expanding around the singularities $u_{D}=\eta_{1}$ and $u_{D}=\eta_{2}$, we see that equation (A1) takes the form

$$
\frac{d^{2}}{d u_{D}^{2}}\left(\delta b_{x}\right)+P(x) \frac{d}{d u_{D}}\left(\delta b_{x}\right)+Q(x) \delta b_{x}=0
$$

where $P(x)=\lambda_{5} x+\lambda_{4}, Q(x)=m_{1} x$ for $u_{D}=\eta_{1}$ and $P(x)=\lambda_{7} x+\lambda_{6}, Q(x)=m_{2} x$ for $u_{D}=\eta_{2}$. By substituting the Taylor expansion of $\delta b_{x}(x, \alpha)$ about the singular point 
$u_{D}=\eta_{1}$ into $(\mathrm{A} 3)$, we get

$$
\begin{gathered}
\frac{d \delta b_{x}}{d u_{D}}=\sum_{n=0}^{\infty} a_{n}(n+\alpha) x^{n+\alpha-1}, \frac{d^{2} \delta b_{x}}{d u_{D}^{2}}=\sum_{n=0}^{\infty} a_{n}(n+\alpha)(n+\alpha-1) x^{n+\alpha-2} \\
\sum_{n=0}^{\infty} a_{n}(n+\alpha)(n+\alpha-1) x^{n+\alpha-2}+\left(\lambda_{5} x+\lambda_{4}\right) \sum_{n=0}^{\infty} a_{n}(n+\alpha) x^{n+\alpha-1} \\
+\left(m_{1} x\right) \sum_{n=0}^{\infty} a_{n} x^{n+\alpha}=0 \\
\sum_{n=0}^{\infty} a_{n}(n+\alpha)(n+\alpha-1) x^{n+\alpha-2}+\lambda_{5} \sum_{n=0}^{\infty} a_{n}(n+\alpha) x^{n+\alpha} \\
+\lambda_{4} \sum_{n=0}^{\infty} a_{n}(n+\alpha) x^{n+\alpha-1}+m_{1} \sum_{n=0}^{\infty} a_{n} x^{n+\alpha+1}=0 \\
\sum_{n=0}^{\infty} a_{n}(n+\alpha)(n+\alpha-1) x^{n+\alpha-2}=a_{0} \alpha(\alpha-1) x^{\alpha-2}+a_{1}(\alpha+1) \alpha x^{\alpha-1} \\
+a_{2}(2+\alpha)(1+\alpha) x^{\alpha}+\sum_{n=0}^{\infty} a_{n+3}(n+\alpha+3)(n+\alpha+2) x^{n+\alpha+1} \\
\sum_{n=0}^{\infty} a_{n}(n+\alpha) x^{n+\alpha}=a_{0} \alpha x^{\alpha}+\sum_{n=0}^{\infty} a_{n+1}(n+\alpha+1) x^{n+\alpha+1} \\
+\alpha) x^{n+\alpha-1}=a_{0} \alpha x^{\alpha-1}+a_{1}(1+\alpha) x^{\alpha}+\sum_{n=0}^{\infty} a_{n+2}(n+\alpha+2) x^{n+\alpha+1}
\end{gathered}
$$

Substitution of (A5), (A6), and (A7) into (A4) and rearrangement of terms yields

$$
\begin{aligned}
a_{0} \alpha(\alpha-1) x^{\alpha-2}+ & {\left[a_{0} \lambda_{4}+a_{1}(\alpha+1)\right] \alpha x^{\alpha-1}+\left[a_{1}(1+\alpha) \lambda_{4}+a_{2}(2+\alpha)(1+\alpha)+a_{0} \alpha \lambda_{5}\right] x^{\alpha} } \\
+ & \sum_{n=0}^{\infty}\left[m_{1} a_{n}+(n+\alpha+1) \lambda_{5} a_{n+1}\right. \\
& \left.+(n+\alpha+2) \lambda_{4} a_{n+2}+(n+\alpha+2)(n+\alpha+3) a_{n+3}\right] x^{n+\alpha+1}=0 .
\end{aligned}
$$

By setting the coefficients of $x^{\alpha-2}, x^{\alpha-1}, x^{\alpha}, x^{n+\alpha+1}$ equal to zero we can find the values of $\alpha$ and we can derive the recursion relation

$$
\begin{gathered}
a_{0} \alpha(\alpha-1)=0 \quad: x^{\alpha-2}, \\
{\left[a_{0} \lambda_{4}+a_{1}(\alpha+1)\right] \alpha=0 \quad: x^{\alpha-1},} \\
a_{1}(1+\alpha) \lambda_{4}+a_{2}(2+\alpha)(1+\alpha)+a_{0} \alpha \lambda_{5}=0 \quad: x^{\alpha}, \\
m_{1} a_{n}+(n+\alpha+1) \lambda_{5} a_{n+1}+(n+\alpha+2) \lambda_{4} a_{n+2}+(n+\alpha+2)(n+\alpha+3) a_{n+3}=0 .
\end{gathered}
$$

By assumption $a_{0} \neq 0$; therefore $\alpha(\alpha-1)=0$, which implies $\alpha_{1}=0, \alpha_{2}=1$. From $\left[a_{0} \lambda_{4}+a_{1}(\alpha+1)\right] \alpha=0$, we conclude that there are two cases to be considered.

Case 1) $\alpha=0$; then $a_{1}$ is arbitrary; and

Case 2) $\alpha=1$; then $a_{1}=-\frac{a_{0} \lambda_{4}}{2}$. 
From $a_{1}(1+\alpha) \lambda_{4}+a_{2}(2+\alpha)(1+\alpha)+a_{0} \alpha \lambda_{5}=0$ we conclude that $a_{2}=-\frac{a_{1}(1+\alpha) \lambda_{4}+a_{0} \alpha \lambda_{5}}{(2+\alpha)(1+\alpha)}$. The recursion relationship can be written as

$$
a_{n+3}=\frac{m_{1} a_{n}+(n+\alpha+1) \lambda_{5} a_{n+1}+(n+\alpha+2) \lambda_{4} a_{n+2}}{(n+\alpha+2)(n+\alpha+3)}, n=0,1,2, \ldots
$$

There are two linearly independent solutions of (A3) around the singular point $u_{D}=\eta_{1}$ in Frobenius form (Bender and Orzag, 1978). Consider the two cases separately:

$$
\begin{aligned}
& \alpha=0, \\
& a_{0} \neq 0, \text { by assumption, } \\
& a_{1} \text { is arbitrary, } \\
& a_{2}=-\frac{a_{1}(1+\alpha) \lambda_{4}+a_{0} \alpha \lambda_{5}}{(2+\alpha)(1+\alpha)}=-\frac{a_{1} \lambda_{4}}{2} \text { when } \alpha=0 .
\end{aligned}
$$

The recursion relationship for $\alpha=0$ is

$$
a_{n+3}=-\frac{m_{1} a_{n}+(n+1) \lambda_{5} a_{n+1}+(n+2) \lambda_{4} a_{n+2}}{(n+2)(n+3)} .
$$

Setting $n=0,1,2,3, \ldots$, we can calculate $a_{3}, a_{4}, \ldots$ :

$$
\begin{aligned}
& n=0: a_{3}=-\frac{m_{1} a_{0}+\lambda_{5} a_{1}+2 \lambda_{4} a_{2}}{6}=-\frac{m_{1} a_{0}+\lambda_{5} a_{1}-\lambda_{4}^{2} a_{1}}{6}=-\frac{m_{1} a_{0}\left(\lambda_{4}^{2}-\lambda_{5}\right) a_{1}}{6} \\
& n=1: a_{4}=-\frac{m_{1} a_{1}+2 \lambda_{5} a_{2}+3 \lambda_{4} a_{3}}{12}=-\frac{1}{24}\left(\left[2 m_{1}+2 \lambda_{4} \lambda_{5}-\lambda_{4}\left(\lambda_{4}^{2}-\lambda_{5}\right)\right] a_{1}+\lambda_{4} m_{1} a_{0}\right) .
\end{aligned}
$$

For $\alpha=0$, we have a solution of Frobenius form:

$$
\delta b_{x}(x, 0)=\sum_{n=0}^{\infty} a_{n} x^{n}=a_{0}+a_{1} x+a_{2} x^{2}+a_{3} x^{3}+\ldots
$$

Substituting the values of $a_{1}, a_{2}, a_{3}$, the solution corresponding to $\alpha=0$ takes the form:

$$
\delta b_{x}(x, 0)=a_{0}\left[1-\frac{m_{1}}{6} x^{3}+\ldots\right]+a_{1}\left[x-\frac{\lambda_{4}}{2} x^{2}+\frac{1}{6}\left(\lambda_{4}^{2}-\lambda_{5}\right) x^{3}+\ldots\right] .
$$

We consider the case when $\alpha=1$ next:

$$
\begin{aligned}
\alpha & =1, \\
a_{0} & \neq 0, \text { by assumption, } \\
a_{1} & =-\frac{a_{0} \lambda_{4}}{2} \\
a_{2} & =-\frac{a_{1}(1+\alpha) \lambda_{4}+a_{0} \alpha \lambda_{5}}{(2+\alpha)(1+\alpha)}=-\frac{2 a_{1} \lambda_{4}+a_{0} \lambda_{5}}{6}=\frac{1}{6}\left(\lambda_{4}^{2}-\lambda_{5}\right) a_{0} \text { when } \alpha=1 .
\end{aligned}
$$

The recursion relationship for $\alpha=1$ is

$$
a_{n+3}=-\frac{m_{1} a_{n}+(n+2) \lambda_{5} a_{n+1}+(n+3) \lambda_{4} a_{n+2}}{(n+3)(n+4)} .
$$


Setting $n=0,1,2,3, \ldots$, we can calculate $a_{3}, a_{4}, \ldots$ :

$$
\begin{aligned}
& n=0: a_{3}=-\frac{m_{1} a_{0}+2 \lambda_{5} a_{1}+3 \lambda_{4} a_{2}}{12}=-\frac{1}{12}\left[m_{1}-\lambda_{4} \lambda_{5}+\frac{1}{2} \lambda_{4}\left(\lambda_{4}^{2}-\lambda_{5}\right)\right] a_{0} \\
& n=1: a_{4}=-\frac{m_{1} a_{1}+3 \lambda_{5} a_{2}+4 \lambda_{4} a_{3}}{20}=\frac{1}{20}\left[\frac{5}{6} m_{1} \lambda_{4}+\frac{1}{6}\left(\lambda_{4}^{2}-3 \lambda_{5}\right)\left(\lambda_{4}^{2}-\lambda_{5}\right)-\frac{1}{3} \lambda_{5} \lambda_{4}^{2}\right] a_{0} .
\end{aligned}
$$

For $\alpha=1$, we have a solution of Frobenius form:

$$
\delta b_{x}(x, 1)=a_{0}+a_{1} x+a_{2} x^{2}+a_{3} x^{3}+\ldots .
$$

Substituting the values of $a_{1}, a_{2}, a_{3}$, the solution corresponding to $\alpha=1$ takes the form:

$$
\begin{aligned}
\delta b_{x}(x, 1)=a_{0}[1 & -\frac{\lambda_{4}}{2} x+\frac{1}{6}\left(\lambda_{4}^{2}-\lambda_{5}\right) x^{2}-\frac{1}{12}\left[m_{1}-\lambda_{4} \lambda_{5}+\frac{1}{2} \lambda_{4}\left(\lambda_{4}^{2}-\lambda_{5}\right)\right] x^{3} \\
& \left.+\frac{1}{20}\left[\frac{5}{6} m_{1} \lambda_{4}+\frac{1}{6}\left(\lambda_{4}^{2}-3 \lambda_{5}\right)\left(\lambda_{4}^{2}-\lambda_{5}\right)-\frac{1}{3} \lambda_{5} \lambda_{4}^{2}\right] x^{4}+\ldots\right] .
\end{aligned}
$$

The general solution to (A3) about $u_{D}=\eta_{1}$ is a linear combination of $\delta b_{x}(x, 0)$ and $\delta b_{x}(x, 1)$.

The structure of the singularity at $u_{D}=\eta_{2}$ is very similar to that of the structure about $u_{D}=\eta_{1}$. The only difference would be to substitute $\lambda_{6}, \lambda_{7}$, and $m_{2}$ for $\lambda_{4}, \lambda_{5}$, and $m_{1}$ respectively. Doing so gives at $\alpha=0$

$$
\delta b_{x}(x, 0)=a_{0}\left[1-\frac{m_{2}}{6} x^{3}+\ldots\right]+a_{1}\left[x-\frac{\lambda_{6}}{2} x^{2}+\frac{1}{6}\left(\lambda_{6}^{2}-\lambda_{7}\right) x^{3}+\ldots\right],
$$

and at $\alpha=1$

$$
\begin{aligned}
\delta b_{x}(x, 1)=a_{0}[1 & -\frac{\lambda_{6}}{2} x+\frac{1}{6}\left(\lambda_{6}^{2}-\lambda_{7}\right) x^{2}-\frac{1}{12}\left[m_{2}-\lambda_{6} \lambda_{7}+\frac{1}{2} \lambda_{6}\left(\lambda_{6}^{2}-\lambda_{7}\right)\right] x^{3} \\
& \left.+\frac{1}{20}\left[\frac{5}{6} m_{2} \lambda_{6}+\frac{1}{6}\left(\lambda_{6}^{2}-3 \lambda_{7}\right)\left(\lambda_{6}^{2}-\lambda_{7}\right)-\frac{1}{3} \lambda_{7} \lambda_{6}^{2}\right] x^{4}+\ldots\right] .
\end{aligned}
$$

The general solution to (A3) about $u_{D}=\eta_{2}$ is a linear combination of $\delta b_{x}(x, 0)$ and $\delta b_{x}(x, 1)$.

Following a similar argument for the singularity at $u_{D}=r \sqrt{\gamma}$ we outline the results here. We substitute the series

$$
\frac{d \delta b_{x}}{d u_{D}}=\sum_{n=0}^{\infty} a_{n}(n+\alpha) x^{n+\alpha-1}, \quad \frac{d^{2} \delta b_{x}}{d u_{D}^{2}}=\sum_{n=0}^{\infty} a_{n}(n+\alpha)(n+\alpha-1) x^{n+\alpha-2},
$$

into (A2) where $x=u_{D}-r \sqrt{\gamma}$ and rearrange terms. Equating the coefficients to zero gives

$$
\alpha=0, \quad \alpha=1-\frac{\epsilon C M T^{2}}{2 \lambda_{1} \lambda_{2}} .
$$

For the case of $\alpha=0$, we find that

$$
a_{1}=0, \quad a_{2}=-\frac{K_{z}^{2} \lambda_{2} T^{2}}{\lambda_{2}\left(2 \lambda_{1} \lambda_{2}+\epsilon C M T^{2}\right)} a_{0} .
$$


Therefore the solution corresponding to $\alpha=0$, about $u_{D}=r \sqrt{\gamma}$, takes the form:

$$
\delta b_{x}(x, 0)=a_{0}\left[1-\frac{K_{z}^{2} \lambda_{2} T^{2}}{\lambda_{2}\left(2 \lambda_{1} \lambda_{2}+\epsilon C M T^{2}\right)} x^{2}+\ldots\right] .
$$

For the case of $\alpha=1-\frac{\epsilon C M T^{2}}{2 \lambda_{1} \lambda_{2}}$, we find that

$$
a_{1}=\frac{\epsilon C M r \sqrt{\gamma}\left(\lambda_{1}+\beta T^{2}\right)\left(\epsilon C M T^{2}-2 \lambda_{1} \lambda_{2}\right)}{\lambda_{1} \lambda_{2} \lambda_{3}\left(4 \lambda_{1} \lambda_{2}-\epsilon C M T^{2}\right)} a_{0} .
$$

Therefore the solution corresponding to $\alpha=1-\frac{\epsilon C M T^{2}}{2 \lambda_{1} \lambda_{2}}$, about $u_{D}=r \sqrt{\gamma}$, takes the form:

$$
\begin{aligned}
& \delta b_{x}\left(x, 1-\frac{\epsilon C M T^{2}}{2 \lambda_{1} \lambda_{2}}\right) \\
& \quad=a_{0}\left[x^{1-\frac{\epsilon C M T^{2}}{2 \lambda_{1} \lambda_{2}}}+\frac{\epsilon C M r \sqrt{\gamma}\left(\lambda_{1}+\beta T^{2}\right)\left(\epsilon C M T^{2}-2 \lambda_{1} \lambda_{2}\right)}{\lambda_{1} \lambda_{2} \lambda_{3}\left(4 \lambda_{1} \lambda_{2}-\epsilon C M T^{2}\right)} x^{2-\frac{\epsilon C M T^{2}}{2 \lambda_{1} \lambda_{2}}}\right] .
\end{aligned}
$$

The general solution to (A2) about $u_{D}=r \sqrt{\gamma}$ is a linear combination of $\delta b_{x}(x, 0)$ and $\delta b_{x}\left(x, 1-\frac{\epsilon C M T^{2}}{2 \lambda_{1} \lambda_{2}}\right)$.

\section{REFERENCES}

Abraham-Shrauner, B., Small amplitude hydromagnetic waves for a plasma with a generalized polytrope law, Plasma Phys. 15, 375-385 (1973)

Aref, H. and Siggia, E. D., Evolution and breakdown of a vortex street in two dimensions, Journal of Fluid Mechanics 109, 435-463 (1981)

Aref, H. and Tryggvason, G., Dynamics of passive and active interfaces, Physica 2D, 59-70 (1984)

Begelman, M. C., Blandford, R. D., and Rees, M. J., Theory of extragalactic radio sources, Review of Modern Physics 56, 255-351 (1984)

Bender, C. M. and Orzag, S. (1978), "Advanced Mathematical Methods for Scientists and Engineers." New York: McGraw-Hill

Blandford, R. D. and Pringle, J. E., Kelvin-Helmholtz instability of relativistic beams, Monthly Notices Roy. Astron. Soc. 176, 443-454 (1976)

Blumen, W., Shear layer instability of an inviscid compressible fluid, Journal of Fluid Mechanics 40, 769-781 (1970)

Blumen, W., Drazin, P. G., and Billings, D. F., Shear layer instability of an inviscid compressible fluid, Part 2, Journal of Fluid Mechanics 71, 305-316 (1975)

Brandt, J. C. and Mendis, D. A. (1979), The solar wind in "Solar System Plasma Physics" (C. F. Kennel, L. Lanzerotti, and E. N. Parker, Eds.), Amsterdam: North Holland

Bridge, H. S., Belcher, J. W., Lazarus, A. J., Sullivan, J. D., Bagenal, F., McNutt, Jr., R. L., Oglivie, K. W., Scudder, J. D., and Sittler, E. C., Plasma observations near Jupiter: Initial results, Science 206, 972-976 (1979)

Brown, G. L. and Roshko, A., On density effects and large structure in turbulent mixing layers, Journal of Fluid Mechanics 64, 775-816 (1974)

Brown, K. G. and Roy Choudhury, S., Kelvin-Helmholtz instabilities of high velocity magnetized shear layers with generalized polytrope laws, Quarterly of Applied Math., 58, 401-423 (2000)

Brown, K. G. and Roy Choudhury, S., An analytical study of the Kelvin-Helmholtz instabilities of compressible, magnetized tangential velocity discontinuities with generalized polytrope laws, Quarterly of Applied Math., to appear

Case, K. M., Stability of inviscid plane Couette flow, Physics of Fluids 3, 143-148; and Stability of an idealized atmosphere I. Discussion of results, Physics of Fluids 3, 149-157 (1960)

Chandrasekhar, S. (1981), "Hydrodynamic and Hydromagnetic Stability." New York: Dover (originally published 1961, Oxford Univ. Press)

Dobrowolny, H. and D'Angelo, N., Wave motion in type I comet tails, in Cosmic Plasma Physics (I. Schindler, ed.), Plenum, New York, 1972 
Duhau, S., Gratton, F., and Gratton, J., Hydromagnetic oscillations of a tangential discontinuity in the CGL approximation, Phys. Fluids 13, 1503-1509 (1970)

Duhau, S., Gratton, F., and Gratton, J., Radiation of hydromagnetic waves from a tangential velocity discontinuity, Phys. Fluids 14, 2067-2071 (1971)

Duhau, S. and Gratton, J., Effect of compressibility on the stability of a vortex sheet in an ideal magnetofluid, Phys. Fluids 16, 150-152 (1972)

Ershkovich, A. I., Nusnov, A. A., and Chernikov, A. A., Oscillations of type I comet tails, Planetary and Space Science 20, 1235-1243 (1972)

Ershkovich, A. I. and Chernikov, A. A., Nonlinear waves in type I comet tails, Planetary and Space Science 21, 663-673 (1973)

Fejer, J. A., Hydromagnetic stability at a fluid velocity discontinuity between compressible fluids, Physics of Fluids 7, 499-503 (1964)

Gerwin, R. A., Stability of the interface between two fluids in relative motion, Rev. Modern Phys. 40, $652-658$ (1968)

Ince, E. L. (1956), "Ordinary Differential Equations." New York: Dover

Jokipii, J. R. and Davis, L., Long wavelength turbulence and the heating of the solar wind, Astrophys. Journal 156, 1101-1106 (1969)

Kaup, D. J., Coherent structures in the planar magnetron, Physics of Fluids B2, 2253-2258 (1990)

Krall, N. A. and Trivelpiece, A. W. (1973), "Principles of Plasma Physics." New York: McGraw-Hill

Landau, L. D., The instability of moving superposed fluids, Akad. Nauk SSSR, Comptes Rendus (Doklady) 44, 139-144 (1944)

Landau, L. D. and Lifshitz, E. M. (1984), "Electrodynamics of Continuous Media." Oxford, Pergamon, $242-243$

Larosa, T. N. and Moore, R. L., A mechanism for bulk energization in the impulsive phase of solar flares, Astrophysical Journal 418, 912-918 (1993)

Lerche, I., Validity of the hydromagnetic approach in discussing instability of the magnetospheric boundary, Journal of Geophys. Res. 71, 2365-2371 (1966)

Michalke, A., On the inviscid instability of the hyperbolic-tangent velocity profile, Journal of Fluid Mechanics 19, 543-556 (1964)

Miles, J. W., On wind over water, Journal Acoust. Soc. Amer. 29, 226-230 (1957)

Miles, J. W., On the disturbed motion of a plane vortex sheet, Journal of Fluid Mechanics 4, 538-552 (1958)

Miura, A. and Pritchett, P. L., Nonlocal stability analysis of the MHD Kelvin-Helmholtz instability in a compressible plasma, J. Geophysical Res. 87, 7431-7444 (1982)

Miura, A., Anomalous transport by magnetohydrodynamic Kelvin-Helmholtz instabilities in the solar wind-magnetosphere interaction, J. Geophysical Res. 89, 801-818 (1984)

Nepveu, M., Cylindrical jets, Astronom. and Astrophys. 84, 14-21 (1980)

Ness, N. F., Acuna, M. H., Lepping, R. P., Connerney, J. E. P., Behannon, K. W., and Burlaga, L. F., Magnetic field studies by Voyager I, Science 212, 211-217 (1981)

Norman, M. L., Smarr, L., Winkler, K. H. A., and Smith, M. D., Instabilities of cylindrical jets, Astronomy and Astrophysics 113, 285-351 (1982)

Parker, E. N. (1963), "Interplanetary Dynamical Processes." New York: Interscience

Pritchett, P. L. and Coroniti, F. V., The collisionless macroscopic Kelvin-Helmholtz instability I. Transverse electrostatic mode, Journal Geophys. Res. 89, 168-178 (1984)

$\mathrm{Pu}, \mathrm{Z}$. Y., Kelvin-Helmholtz instability in collisionless space plasmas, Phys. Fluids B 1, $440-447$ (1989) $\mathrm{Pu}, \mathrm{Z}$. Y. and Kivelson, M. G., Kelvin-Helmholtz instability at the magnetopause: Solution for compressible plasmas, Journal Geophys. Res. 88, 841-852; and, Energy flux into the magnetosphere, Journal Geophys. Res. 88, 853-861 (1983)

Rajaram, R., Kalra, G. L., and Tandon, J. N., Discontinuities and the magnetospheric phenomena, J. Atm. Terr. Phys. 40, 991-1000 (1978)

Rajaram, R., Kalra, G. L., and Tandon, J. N., Discontinuities in the magnetosphere, Astrophys. Space Sci. 67, 137-150 (1980)

Ray, T. P., The effects of a simple shear layer on the growth of Kelvin-Helmholtz instabilities, Monthly Notices Roy. Astronom. Soc. 198, 617-625 (1982)

Ray, T. P. and Erschkovich, A. I., Kelvin-Helmholtz instabilities of magnetized shear layers, Monthly Notices Roy. Astron. Soc. 204, 821-826 (1983) 
Roy Choudhury, S. and Lovelace, R. V. E., On the Kelvin-Helmholtz instabilities of supersonic shear layers, Astrophysical J. 283, 331-342 (1984)

Roy Choudhury, S. and Lovelace, R. V. E., On the Kelvin-Helmholtz instabilities of high-velocity magnetized shear layers, Astrophysical J. 302, 188-199 (1986)

Roy Choudhury, S., Kelvin-Helmholtz instabilities of supersonic, magnetized shear layers, J. Plasma Phys. 35, 375-392 (1986)

Roy Choudhury, S. and Patel, V. L., Kelvin-Helmholtz instabilities of high-velocity, magnetized anisotropic shear layers, Phys. Fluids 28, 3292-3301 (1985)

Roy Choudhury, S., Global asymptotic analysis of the Kelvin-Helmholtz instability of supersonic shear layers, Canadian J. Physics 68, 334-342 (1990)

Sen, A. K., Stability of the magnetosphere boundary, Planetary and Space Science 13, 131-141 (1965)

Sen, A. K., Effect of compressibility on Kelvin-Helmholtz instability in a plasma, Physics of Fluids $\mathbf{7}$, 1293-1298 (1964)

Southwood, D. J., The hydromagnetic stability of the magnetospheric boundary, Planetary and Space Science 16, 587-605 (1968)

Southwood, D. J., Some features of the field line resonances in the magnetosphere, Planetary and Space Science 22, 483-491 (1974)

Sturrock, P. A. and Hartle, R. E., Two-fluid mode of the solar wind, Physical Review Lett. 16, 628-631 (1966)

Syrovatskii, A., The Helmholtz instability, Soviet Physics Uspekhi 62, 247-253 (1957)

Tajima, T. and Leboeuf, J. N., Kelvin-Helmholtz instability in supersonic and super-Alfvénic fluids, Physics of Fluids 23, 884-888 (1980)

Talwar, S. P., Hydromagnetic stability of the magnetospheric boundary, J. Geophysical Res. 69, 27072713 (1964)

Talwar, S. P., Kelvin-Helmholtz instability in an anisotropic plasma, Phys. Fluids 8, 1295-1299 (1965)

Turland, B. D. and Scheuer, P. A. G., Instabilities of Kelvin-Helmholtz type for relativistic streaming, Monthly Notices Roy. Astron. Soc. 176, 421-441 (1976)

Winant, C. D. and Browand, F. K., Vortex pairing: The mechanism of turbulent mixing-layer growth, Journal Fluid Mech. 63, 237-255 (1974) 